\title{
Protective effects of aucubin on osteoarthritic chondrocyte model induced by hydrogen peroxide and mechanical stimulus
}

In-Chi Young ${ }^{1}$, Sung-Ting Chuang ${ }^{2}$, Chia-Hsien Hsu ${ }^{3}$ Yu-Jun Sun ${ }^{1}$, Hwa-Chang Liu ${ }^{4,5}$, Yo-Shen Chen ${ }^{1}$ and Feng-Huei Lin ${ }^{1 *}$

\begin{abstract}
Background: During the onset of osteoarthritis $(\mathrm{OA})$, certain biochemical events have been shown to accelerate cartilage degradation, including the dysregulation of cartilage ECM anabolism, abnormal generation of reactive oxygen species (ROS) and overproduction of proteolytic enzymes and inflammatory cytokines. The potency of aucubin in protecting cellular components against oxidative stress, inflammation and apoptosis effects are well documented, which makes it a potential candidate for OA treatment. In this study, we aimed to evaluate the protective benefits of aucubin against $\mathrm{OA}$ using $\mathrm{H}_{2} \mathrm{O}_{2}$ and compression induced $\mathrm{OA}$-like chondrocyte models.
\end{abstract}

Methods: The effects of aucubin were studied in porcine chondrocytes after $1 \mathrm{mM} \mathrm{H}_{2} \mathrm{O}_{2}$ stimulation for 30 min or sustained compression for $24 \mathrm{~h}$. Effects of aucubin on cell proliferation and cytotoxicity of chondrocytes were measured with WST-1 and LDH assays. ROS production was evaluated by the Total ROS/Superoxide Detection Kit. Caspase-3 activity was evaluated by the CaspACE assay system. The levels of apoptosis were evaluated by the Annexin V-FITC apoptosis detection kit. OA-related gene expression was measured by reverse transcription quantitative polymerase chain reaction (RT-qPCR). Total DNA quantification was evaluated by the DNeasy Blood and Tissue kit. Sulfated-glycosaminoglycans (sGAGs) production and content were evaluated by DMMB assay and Alcian blue staining.

Results: The results showed that the ROS scavenge effects of aucubin appeared after $1 \mathrm{~h}$ of pretreatment. Aucubin could reduce the caspase-3 activity induced by $\mathrm{H}_{2} \mathrm{O}_{2}$, and reduced the apoptosis cell population in flowcytometry. In RT-qPCR results, aucubin could maintain ACAN and COL2A1 gene expressions, and prevent IL6 and MMP13 gene upregulation induced by $\mathrm{H}_{2} \mathrm{O}_{2}$ and compression stimulations. In the DMMB assay and Alcian blue staining, aucubin could maintain the sGAG content and protect chondrocytes against compressive stress, but not oxidative stress from $\mathrm{H}_{2} \mathrm{O}_{2}$.

Conclusions: These results indicated that aucubin has protective effects in an osteoarthritic chondrocyte model induced by $\mathrm{H}_{2} \mathrm{O}_{2}$ and mechanical stimulus.

Keywords: Aucubin, Osteoarthritis, ROS, Mechanical stress, Inflammation

\footnotetext{
* Correspondence: double@ntu.edu.tw

${ }^{1}$ Institute of Biomedical Engineering, National Taiwan University, No. 49,

Fanglan Rd, Taipei 10672, Taiwan

Full list of author information is available at the end of the article
} 


\section{Background}

Osteoarthritis (OA) is the most common form of arthritis and the leading cause of disability in people over 65 years old $[1,2]$. The high prevalence rate among the elderly makes it a considerable clinical and economic burden because of reduced quality of life and increased use of health care resources [3, 4]. OA involves the entire joint, including the subchondral bone, ligaments, periarticular muscle and synovium, and is associated with risk factors, such as age, gender, prior joint injury, obesity, genetic predisposition and mechanical stress $[5,6]$. In healthy individuals, synthesis and degradation of cartilage extracellular matrix (ECM) maintains a particular balance. However, this homeostatic balance can be disrupted in OA cartilage by reduced anabolic and increased catabolic capacities of chondrocytes. Chondrocytes are the unique cells of the articular cartilage ECM and are responsible for the synthesis and degradation of the cartilage, which mainly consists of type II collagen and sulfated proteoglycans (sGAG) [7]. During the initiation of OA, biochemical events have been shown to accelerate the cartilage degradation, including the dysregulation of the ECM anabolism, abnormal generation of reactive oxygen species (ROS) and overproduction of proteolytic enzymes and inflammatory cytokines [8-10]. Disruption of homeostasis decreases type II collagen and sGAG and leads to the loss of cellularity via apoptosis, which plays a central role in the caspase proteolytic cascade, all of which is evident in the OA cartilage [11].

Damage from mechanical stress with insufficient selfrepair by joints is believed to be the primary cause of OA. Excessive compressive stress can increase the production of ROS in chondrocytes, which is sufficient to depolymerize hyaluronic acid of ECM [12] or even kill chondrocytes [13]. In response to mechanical loading of articular cartilage, chondrocytes exhibit the same changes in gene expression as those during OA, such as upregulation of the matrix metalloproteinase 13 gene (MMP13) and a disintegrin and metalloproteinase with thrombospondin motifs (ADAMTS) gene family. Therefore, cartilage explants stimulated with mechanical stresses have been used as typical experimental models [14].

Several studies have shown that ROS, such as superoxide anions, hydroxyl radicals and hydrogen peroxide $\left(\mathrm{H}_{2} \mathrm{O}_{2}\right)$, play a role in cartilage degeneration $[15,16]$. A high-level of ROS production commonly results in apoptosis and senescence of the chondrocytes, and thus associates with a decreased number of chondrocytes and altered cell phenotypes during $\mathrm{OA}$ [17]. $\mathrm{H}_{2} \mathrm{O}_{2}$ is a potent mediator of membrane lipid peroxidation. The disruption of mitochondrial membrane integrity caused by $\mathrm{H}_{2} \mathrm{O}_{2}$ leads to the release of caspases that play essential roles in apoptosis [18]. Thus, $\mathrm{H}_{2} \mathrm{O}_{2}$ is frequently used as an inducer of oxidative stress to investigate the role of cellular antioxidants, like vitamin E, in ameliorating cellular injury [19].
In our previous studies, we have found that antioxidants from Chinese herbal medicines have potential benefits in long-term treatments of ROS-mediated diseases [20]. Aucubin, an iridoid glucoside isolated from various plants including leaves of Aucuba japonica and Eucommia ulmoides [21, 22], has proven to possess numerous pharmacological effects [23-25]. The potential role of aucubin in protecting cellular components against oxidative stress and inflammatory responses is well documented [26, 27]. However, the effect of aucubin on the catabolic responses of chondrocytes or its therapeutic role in OA has not been identified.

In this study, we investigated the effects of aucubin on the following gene expressions: ECM-related genes (COL2A1 and ACAN), the catabolic MMP13 gene and the proinflammatory cytokine IL6 gene. The effect of aucubin on ROS production, caspase-3 activity, chondrocyte apoptosis, cell proliferation activity and cytotoxicity, sGAG production and sGAG content were also investigated. Finally, we discuss the potential utility of aucubin as a treatment for OA in light of our findings.

\section{Methods}

\section{Isolation of chondrocytes}

The isolation and use of porcine chondrocytes were approved by the Animal Experimentation Ethics Committee of National Taiwan University Hospital. Fresh porcine stifles were purchased from a traditional market and kept integrated till chondrocytes were isolated under aseptic conditions. A total of 12 porcine stifles were used for all experiments. Porcine chondrocytes were isolated from macroscopically normal cartilage of the femoral condyles [28]. Finely diced cartilage pieces were treated with $10 \%$ antibiotics (15240-062, Gibco, USA) in phosphate buffered saline (PBS) at $37{ }^{\circ} \mathrm{C}$ for $10 \mathrm{~min}$, then re-suspended in Dulbecco's modified eagle's medium (DMEM; D5648, Sigma, USA) containing 10\% fetal bovine serum (12003C, SAFC, USA), $1 \%$ penicillin and $0.05 \%$ L-Ascorbic acid (A5960, Sigma, USA) and 0.2\% collagenase (C0130, Sigma, USA) at $37{ }^{\circ} \mathrm{C}$ for $18 \mathrm{~h}$. Chondrocytes were then collected and washed twice with PBS, and cultured in DMEM. Chondrocytes with a passage number of 2 to 4 were used in all experiments.

\section{Cell proliferation and cytotoxicity of aucubin on chondrocytes}

Chondrocytes were seeded in 96-well cell culture plates at a density of $1 \times 10^{4}$ cells per well and cultured in DMEM for $18 \mathrm{~h}$. Cells were then cultured in the medium containing 3, 10, 30, $100 \mu \mathrm{M}$ aucubin (55561, Sigma, USA). To evaluate the cell proliferation activity of aucubin on chondrocytes, WST-1 assay (Cell Proliferation Reagent WST-1; Roche, Germany) was performed on days 1 and 3 . The OD value was measured at $450 \mathrm{~nm}$ 
using the enzyme-linked immunosorbent assay (ELISA) reader (Sunrise, Tecan, Switzerland).

The LDH assay (CytoTox96 Non-Radioactive Cytotoxicity Assay; Promega, USA) was performed on days 1 and 3 to evaluate the cytotoxicity of aucubin. LDH released in the culture supernatants was measured with a 30-min coupled enzymatic assay and measured using the ELISA reader at a wavelength of $490 \mathrm{~nm}$. The percentage of cytotoxicity was calculated using the following equation:

$$
\text { Cytotoxicity }(\%)=\frac{\mathrm{OD}_{\text {exp }}-\mathrm{OD}_{\text {medium }}}{\mathrm{OD}_{\text {total lysis }}-\mathrm{OD}_{\text {medium }}} \times 100
$$

For both WST-1 and LDH assays, Chondrocytes derived from more than three porcine stifles were used for the experiments. Experiments were performed in five repeated measurements.

\section{Aucubin pretreatment and induction of oxidative stress} The chondrocytes were seeded in 6-well cell culture plates with a density of $1 \times 10^{5}$ cells per well, cultured in DMEM with $10 \%$ fetal bovine serum (Gibco, USA) and incubated overnight. Cells were pretreated with $100 \mu \mathrm{M}$ aucubin in full medium for $24 \mathrm{~h}$. After washing once with PBS, the oxidative stress was induced by introducing $1 \mathrm{mM} \mathrm{H}_{2} \mathrm{O}_{2}$ (RDH, USA) in full medium for $30 \mathrm{~min}$, which was followed by a second PBS wash, and then cultured in fresh medium at $37^{\circ} \mathrm{C}$ for $24 \mathrm{~h}$.

\section{ROS scavenge effect}

ROS production was evaluated by Total ROS/Superoxide Detection Kit (ENZ-51010, Enzo Life Sciences, USA). After $\mathrm{H}_{2} \mathrm{O}_{2}$ treatment, chondrocytes were collected, washed twice with PBS and stained with $500 \mu \mathrm{l}$ of the ROS detection mix for $30 \mathrm{~min}$ in the dark, and then analyzed with an ELISA reader (Spectra Max, Molecular Devices, USA). ROS fluorescence was also examined using a confocal laser scanning biological microscope (IX71/FV300, Olympus, Japan). Chondrocytes derived from more than three porcine stifles were used for the experiments. Experiments were performed in five repeated measurements.

\section{Caspase-3 activity}

Chondrocytes were collected after $\mathrm{H}_{2} \mathrm{O}_{2}$ treatment. Total protein content was determined using a BCA protein assay kit (Pierce, USA) according to the manufacturer's instructions. Caspase- 3 activity was evaluated by a CaspACE assay system (Promega, USA). Thirty microgram of protein from each sample was mixed with a reaction buffer containing $2 \mu \mathrm{l}$ of DMSO, $10 \mu \mathrm{l}$ of $100 \mathrm{mM}$ DTT and $32 \mu \mathrm{l}$ of caspase assay buffer in a 96well microplate. Two microlitre of DEVD-pNA was then added and the proteins in solution were incubated at $37{ }^{\circ} \mathrm{C}$ for $4 \mathrm{~h}$. The absorbance was measured at the wavelength of $405 \mathrm{~nm}$ using an ELISA reader (Sunrise, Tecan, Switzerland). Chondrocytes derived from more than three porcine stifles were used for the experiments. Five repeated measurements were performed for all samples.

\section{Chondrocyte apoptosis}

The chondrocyte apoptosis was evaluated by Annexin V-FITC apoptosis detection kit (ab14085, Abcam, USA). At the end of aucubin pretreatment and $\mathrm{H}_{2} \mathrm{O}_{2}$ stimulation, chondrocytes were collected, washed twice with cold PBS and stained with $500 \mu \mathrm{l}$ of the Annexin VFITC and PI mixed solution or $30 \mathrm{~min}$ in the dark, then analyzed by flow cytometry (FC500, Beckman, USA). Chondrocytes derived from more than three porcine stifles were used for the experiments. Five repeated measurements were performed for all samples.

\section{Induction of compressive stress}

The compressive stress was introduced with a custommade compressive device. We incorporated chondrocytes with chitosan-gelatin-glycerol phosphate hydrogel as cell carrier [29]. The hydrogel incorporated chondrocytes were seeded into the wells of the compression device $(200 \mu \mathrm{l} /$ well $)$ and cultured at $37{ }^{\circ} \mathrm{C}$. After $24 \mathrm{~h}$ of incubation, wells with cells were covered with polydimethylsiloxane membrane. The compression was given via nitrogen gas with pressure of $60 \mathrm{psi}$, which compressed the cells through the structural depression of membrane, and chondrocytes were subjected to sustained compression for $24 \mathrm{~h}$. Chondrocytes without treatment were used as control group.

\section{RNA extraction and gene expression of chondrocytes}

The chondrocytes were collected and total RNA was extracted using RNeasy Protect Mini kit (74104, QIAGEN, Germany). Total RNA yield and RNA quality were detected by spectrophotometer (NanoDrop ${ }^{\mathrm{Tm}} 2000$, Thermo Fisher Scientific, USA). RNA samples showed an A260/ 280 ratio between 1.8 2.0 and an A260/230 ratio between 2.0 2.2 were used for reverse transcription quantitative polymerase chain reaction (RT-qPCR). The first strand complementary DNA (cDNA) was synthesized from RNA and SuperScript ${ }^{\mathrm{tm}}$ III First-Strand Synthesis System (18080-051 Invitrogen, USA) according to the instructions provided by the manufacturer. The volume of the PCR Mix of single reaction was $20 \mu$ l containing $1 \mu \mathrm{l}$ of primer solution, $9 \mu \mathrm{l}$ of cDNA and $10 \mu \mathrm{l}$ of $2 \times$ TaqMan Universal PCR Master Mix (4304437, ABI, USA). TaqMan Gene Expression Assays (Life Technology, USA) were used for gene expression analysis. The target genes of RT-qPCR are summarized in Table 1. 
Table 1 Primers used in this study

\begin{tabular}{lll}
\hline Target gene & Assay ID & GeneBank accession number \\
\hline GAPDH & Ss03375435_u1 & AF141959.1 \\
COL2A1 & Ss03373344_g1 & AF201724.1 \\
ACAN & Ss03374824_g1 & X60107.1 \\
MMP13 & Ss03373279_m1 & AF069643.1 \\
IL6 & Ss03384604_u1 & AB194100.1 \\
\hline
\end{tabular}

RT-qPCR was performed using an ABI PRISM 7900HT Sequence Detection System and Sequence Detection Software 2.2.2. The target genes were normalized to the glyceraldehyde-3-phosphate dehydrogenase (GAPDH). The relative mRNA expression of each target gene was determined using the ${ }^{\Delta \Delta} \mathrm{Ct}$ method.

\section{Total DNA quantification}

After compression $/ \mathrm{H}_{2} \mathrm{O}_{2}$ treatment, chondrocytes were collected and total DNA was purified using the DNeasy Blood and Tissue kit (69504, QIAGEN, Germany), following the instructions provided by the manufacturer. Total DNA yield was quantified by an ultra violet/visible/near infrared (UV/VIS/NIR) spectrophotometer (DU 7500, Beckman, USA) at the wavelength of 260 and $280 \mathrm{~nm}$. The ratio of 260 to $280 \mathrm{~nm}$ was between 1.8 and 2.0 .

\section{Analysis of sGAG production}

The sGAG production was evaluated in a DMMB (341088, Sigma, USA) assay, as previously described [29]. At the end of compression $/ \mathrm{H}_{2} \mathrm{O}_{2}$ treatment, cells were reseeded in 6-well cell culture plates and incubated for 3 days. The culture medium of each sample was collected and $40 \mu \mathrm{l}$ of the supernatant of each sample transferred to a 96-well microplate, after which $250 \mu \mathrm{l}$ of DMMB solution was added. The DMMB-sGAG complex product was examined by an ELISA reader (Sunrise, TECAN, Switzerland) at the wavelength of $595 \mathrm{~nm}$. The sGAG production activity of each sample was determined using a calibration curve of condroitin-6-sulfate (C4384, Sigma, USA). The sGAG production was normalized to cell numbers by a total DNA assay (sGAG to DNA ratio). Chondrocytes derived from more than three porcine stifles were used for the experiments. Five repeated measurements were performed for all samples.

\section{Alcian blue staining}

After compression $/ \mathrm{H}_{2} \mathrm{O}_{2}$ treatment, cells were reseeded into 4-well chamber-slides and cultured for 3 days. Chondrocytes were washed twice with PBS and fixed in $10 \%$ neutral buffered formalin (H121-08, Mallinckrodt Analytical, USA) for $30 \mathrm{~min}$ and then washed twice with PBS. Alcian blue (pH 1.0, Muto pure chemicals, Japan) was added for $30 \mathrm{~min}$ and cells were then washed in running water for $1 \mathrm{~min}$. Nuclear fast red (1001210500, Merck, Germany) was added for $5 \mathrm{~min}$ and then washed in running water for $1 \mathrm{~min}$. The cells were dehydrated in 2 changes of $95 \%$ alcohol and absolute alcohol (459844, Sigma, USA) for 1 min each. The sGAG content images were taken by using an IX71 inverted microscope equipped with a DP30BW digital camera system (Olympus, Japan). Chondrocytes derived from more than three porcine stifles were used for the experiments. Five repeated measurements were performed for all samples.

\section{Statistical analysis}

The normality of variance of data was tested before statistical analysis. Statistically significant differences between the groups were determined by one-way ANOVA with Tukey's post-hoc test. The results were expressed as mean \pm standard deviation of the mean (SD) and considered significant when the $P$-value was $<0.05$. Statistical analysis was performed using the SigmaPlot version 12.3 software (Systat Software Inc., San Jose, USA).

\section{Results}

Aucubin shows no cytotoxic effects on chondrocytes

As shown in Fig. 1, chondrocytes treated with 3, 10, 30, and $100 \mu \mathrm{M}$ of aucubin showed no significant differences in cytotoxicity or cell proliferation activity compared to controls at 1 or 3 days, indicating that aucubin within the concentration range of $3-100 \mu \mathrm{M}$ shows no significant cytotoxic effects on chondrocytes at either 1 or 3 days.

\section{Aucubin inhibits $\mathrm{H}_{2} \mathrm{O}_{2}$-induced ROS production in chondrocytes}

As shown in Fig. 2, the ROS production was significantly increased in response to $\mathrm{H}_{2} \mathrm{O}_{2}$ stimulation. Chondrocytes treated with aucubin showed significant ROS scavenging effects after 1,4 and $24 \mathrm{~h}$ of incubation with 10 to $100 \mu \mathrm{M}$ aucubin in a dose-dependent manner. The ROS scavenge effect of aucubin was further confirmed through fluorescence imaging. As shown in Fig. 3, both the green and red fluorescence emissions, representing general ROS and superoxide induced by $\mathrm{H}_{2} \mathrm{O}_{2}$, respectively, were markedly reduced in fluorescence intensity and area after $24 \mathrm{~h}$ of $100 \mu \mathrm{M}$ aucubin pretreatment. Since $100 \mu \mathrm{M}$ aucubin showed the greatest scavenging effect with limited cytotoxicity among other aucubin concentrations, we used $100 \mu \mathrm{M}$ aucubin in the subsequent experiments.

\section{Aucubin inhibits $\mathrm{H}_{2} \mathrm{O}_{2}$-induced caspase-3 activity on chondrocytes}

After $\mathrm{H}_{2} \mathrm{O}_{2}$ treatment, the caspase- 3 activity of chondrocytes showed a significant increase. However, chondrocytes treated with aucubin and caspase inhibitor 


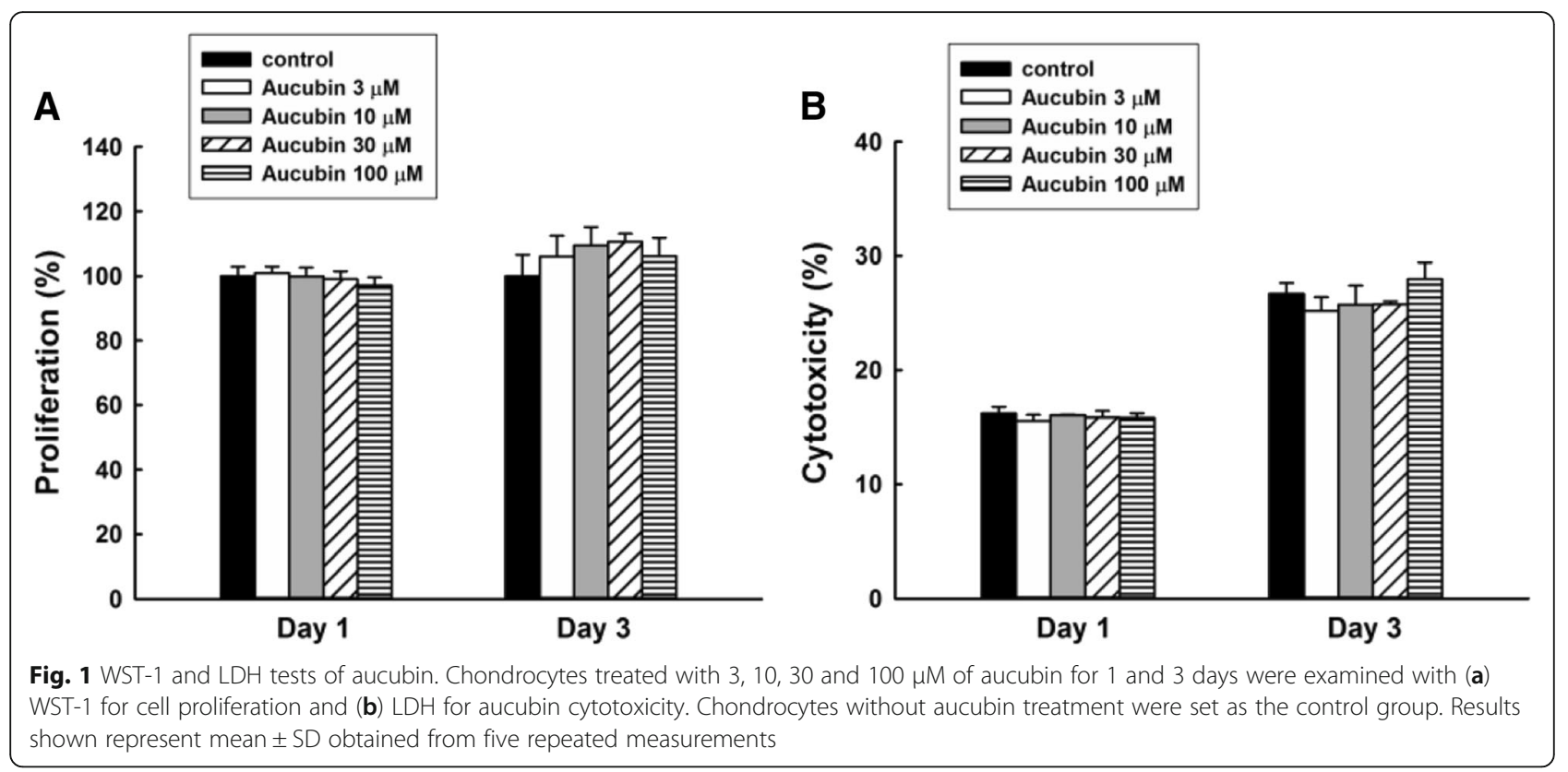

Z-VAD-FMK markedly decreased the caspase-3 activity caused by $\mathrm{H}_{2} \mathrm{O}_{2}$ stimulation (Fig. 4).

\section{Aucubin inhibits $\mathrm{H}_{2} \mathrm{O}_{2}$-induced apoptosis and necrosis in chondrocytes}

After $\mathrm{H}_{2} \mathrm{O}_{2}$ treatment, the percentage of apoptotic and necrotic chondrocytes were significantly increased compared to the control group. However, in the aucubin pretreated group, the percentages of chondrocytes in both early and late apoptosis states were significantly reduced compared to the $\mathrm{H}_{2} \mathrm{O}_{2}$ stimulated group (Fig. 5, Table 2).

\section{Aucubin reverses $\mathrm{H}_{2} \mathrm{O}_{2}$-mediated and compression-mediated} gene expression of ACAN and COL2A1 in chondrocytes

ACAN and COL2A1 are ECM related genes. As shown in the $\mathrm{H}_{2} \mathrm{O}_{2}$ model of Fig. 6a and b, the expression of both ACAN and COL2A1 was significantly down-

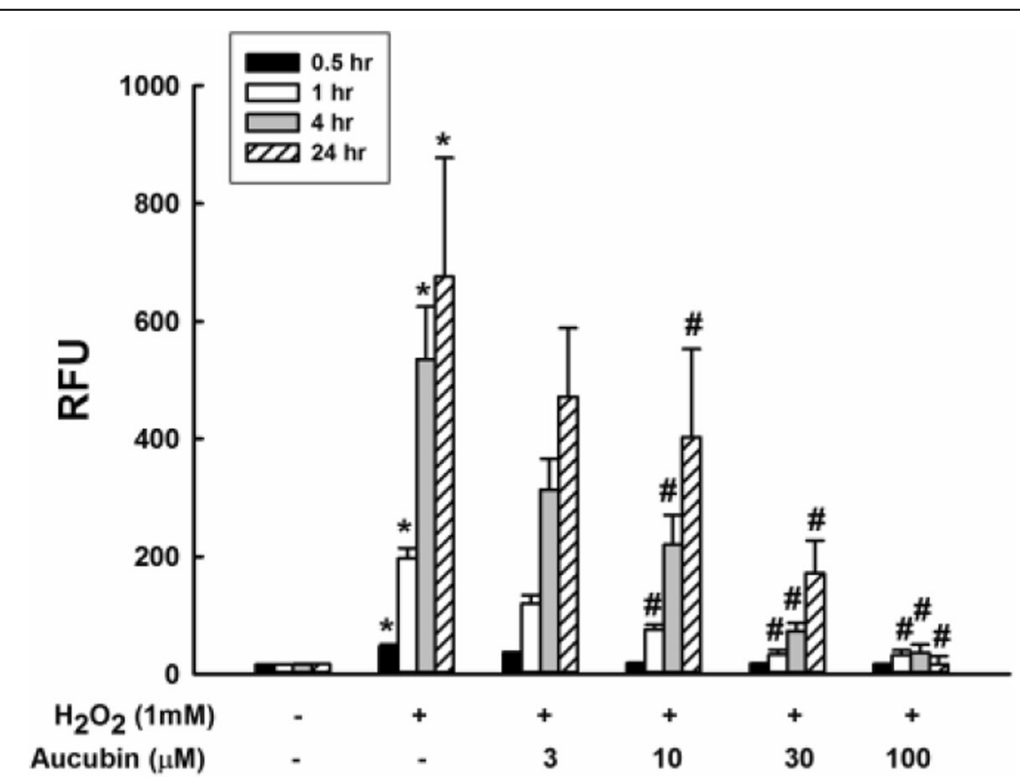

Fig. 2 ROS scavenge test of aucubin. Chondrocytes pretreated with $0,3,10,30$ and $100 \mu \mathrm{M}$ of aucubin for $0.5,1,4$ and $24 \mathrm{~h}$ were stimulated with $1 \mathrm{mM} \mathrm{H}_{2} \mathrm{O}_{2}$ for 30 min. ROS production was measured by the intensity of the dye fluorescence and represented as relative fluorescence unit (RFU). Results shown represent mean \pm SD obtained from five repeated measurements. ${ }^{*} p<0.05$ compared with the control group. ${ }^{\#} p<0.05$ compared with the $\mathrm{H}_{2} \mathrm{O}_{2}$ group 


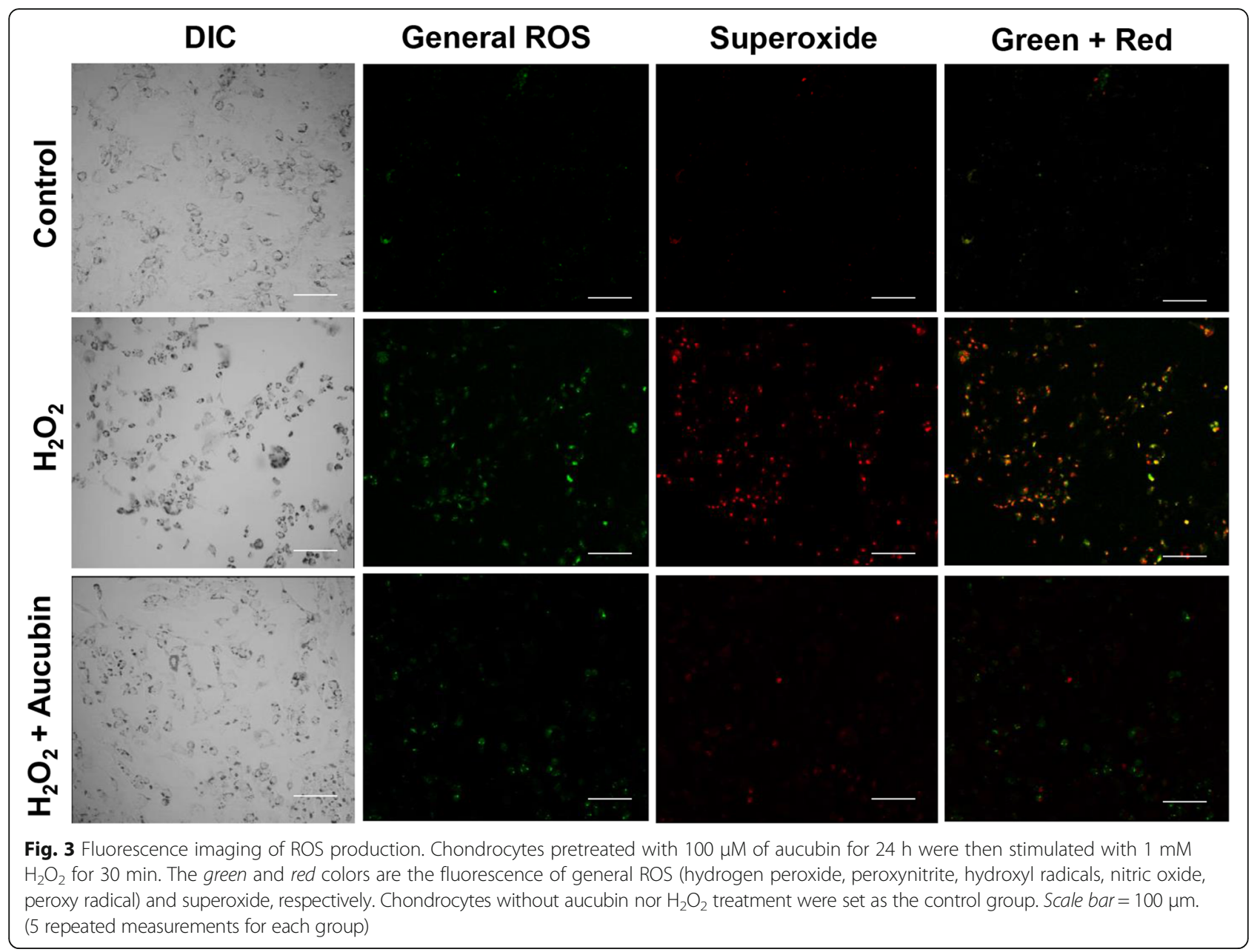

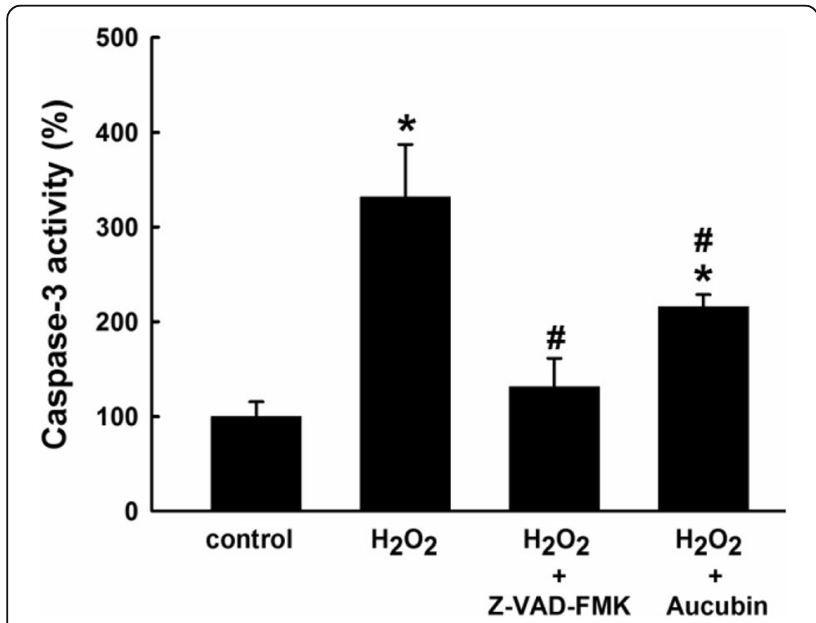

Fig. 4 Effect of aucubin on caspase-3 activity. Chondrocytes pretreated with $100 \mu \mathrm{M}$ of aucubin or Z-VAD-FMK as caspase inhibitor for $24 \mathrm{~h}$ were then stimulated with $1 \mathrm{mM} \mathrm{H}_{2} \mathrm{O}_{2}$ for $30 \mathrm{~min}$. Chondrocytes without aucubin nor $\mathrm{H}_{2} \mathrm{O}_{2}$ treatment were set as the control group. Results shown represent mean \pm SD obtained from five repeated measurements. ${ }^{*} p<0.05$ compared with the control group. ${ }^{*} p<0.05$ compared with the $\mathrm{H}_{2} \mathrm{O}_{2}$ group regulated in the $\mathrm{H}_{2} \mathrm{O}_{2}$-treated group compared to the control group. However, pretreatment with aucubin reversed the expression of these genes back to the level of the control group and showed marked up-regulation compared to the $\mathrm{H}_{2} \mathrm{O}_{2}$-treated group. Similar results were shown in the compression model. The expression of both ACAN and COL2A1 was also markedly downregulated in the loading group, though the aucubin pretreated chondrocytes had a significant up-regulation of both ECM component genes after compression stimulation compared to the control group (Fig. $6 \mathrm{c}$ and d).

\section{Aucubin reduces $\mathrm{H}_{2} \mathrm{O}_{2}$-mediated and compression-mediated} gene expression of MMP13 and IL6 in chondrocytes

MMP13 is a catabolic gene in cartilage ECM homeostasis. As shown in Fig. 6e and g, in both $\mathrm{H}_{2} \mathrm{O}_{2}$ and compression models, the expression of MMP13 was up-regulated compared to the control group after stimulation. However, treatment with aucubin before $\mathrm{H}_{2} \mathrm{O}_{2}$ and compression stimulation maintained the expression of MMP13 at a comparable level to the control group. Similar results were shown in the 

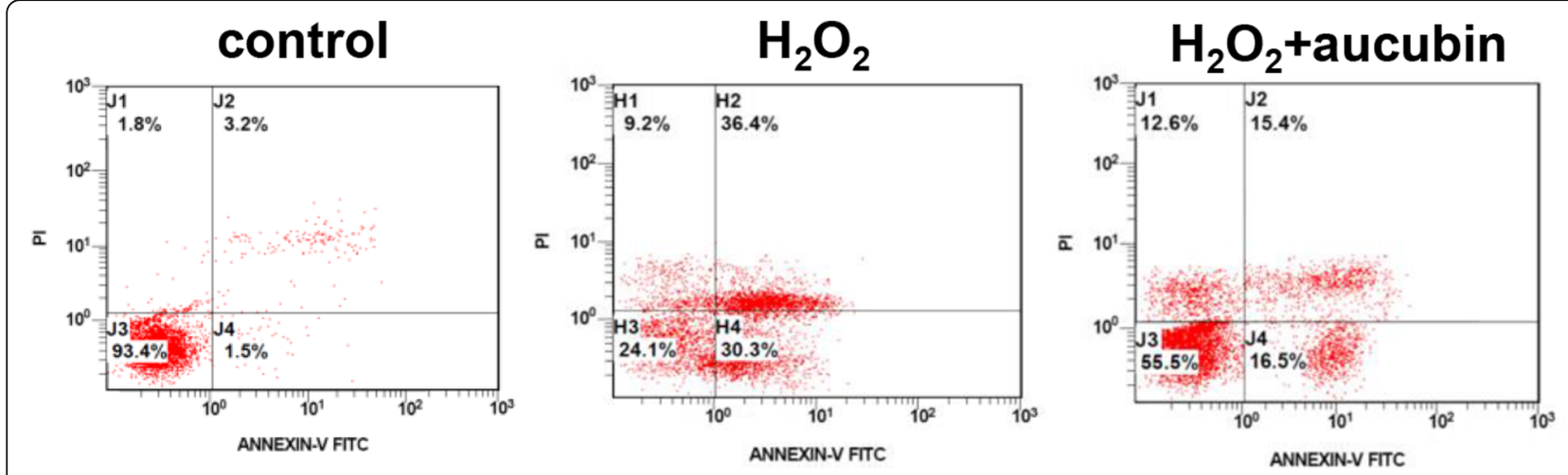

Fig. 5 Annexin V/PI test of aucubin. Chondrocytes pretreated with $100 \mu \mathrm{M}$ of aucubin for $24 \mathrm{~h}$ were then stimulated with 1 mM $\mathrm{H}_{2} \mathrm{O}_{2}$ for $30 \mathrm{~min}$. Chondrocytes without aucubin nor $\mathrm{H}_{2} \mathrm{O}_{2}$ treatment were set as the control group. (5 repeated measurements for each group)

expression of the proinflammatory gene IL6. As shown in Fig. $6 \mathrm{f}$ and h, IL6 was up-regulated compared to the control group in both models after stimulation. Moreover, the up-regulated IL6 expression was reversed to a similar level as the control group with the pretreatment of aucubin.

\section{Aucubin increases sGAG production in compression model but not after $\mathrm{H}_{2} \mathrm{O}_{2}$ stimulation}

As shown in Fig. 7a, the sGAG to DNA ratio for the $\mathrm{H}_{2} \mathrm{O}_{2}$ treated group was significantly decreased compared to the control group. Treatment with aucubin showed no significant difference for the $\mathrm{H}_{2} \mathrm{O}_{2}$ treated group. However, the decreased sGAG to DNA ratio induced by compressive stress was significantly increased compared to the compression group (Fig. 7b).

\section{Aucubin increases the sGAG content after both $\mathrm{H}_{2} \mathrm{O}_{2}$ and compression stimulation}

As shown in Fig. 7c and d, the alcian blue staining was positive in the control groups for both $\mathrm{H}_{2} \mathrm{O}_{2}$ and compression models. The $\mathrm{H}_{2} \mathrm{O}_{2}$ treated group, on the contrary, showed few areas of blue color with shrinking cell morphology. With aucubin pretreatment, some of the chondrocytes could maintain sGAG content. Similar results were also evident in the compression model.

\section{Discussion}

Aucubin has significant antioxidant and radical scavenging properties in both in vivo and in vitro models [30], making it a potential therapeutic agent in oxidative stress-induced diseases. Aucubin has been shown to reduce ROS formation, malondialdehyde levels and $\beta$ galactosidase activity, and increase glutathione levels in UVB-irradiated human skin fibroblasts [31]. To evaluate the protective effects of aucubin in regards to OA, first we generated an osteoarthritic cell model with porcine chondrocytes by using $1 \mathrm{mM} \mathrm{H}_{2} \mathrm{O}_{2}$, which decreased $60 \%$ of chondrocytes proliferation activity but showed no significant or immediate cytotoxicity to untreated control (data not shown). In this study, 10$100 \mu \mathrm{M}$ of aucubin caused no significant cytotoxicity or differences in cell proliferation activity after incubation with chondrocytes at 1 or 3 days (Fig. 1). After $\mathrm{H}_{2} \mathrm{O}_{2}$ stimulation, $10-100 \mu \mathrm{M}$ of aucubin was a sufficient range to reduce ROS production dose-dependently after at least $1 \mathrm{~h}$ of pretreatment (Fig. 2). Pretreatment with $100 \mu \mathrm{M}$ of aucubin also inhibited $\mathrm{H}_{2} \mathrm{O}_{2}$-induced caspase-3 activity (Fig. 4). Xue et al. reported that aucubin inhibited $\mathrm{H}_{2} \mathrm{O}_{2}$-induced apoptosis in $\mathrm{PC} 12$ cells through regulation of the endogenous oxidant-antioxidant balance [27]. In this study, we confirmed the apoptosis inhibition properties of aucubin via AnnexinV/PI flow cytometry (Fig. 5). The percentage of apoptotic cells was significantly reduced when $100 \mu \mathrm{M}$ of aucubin were used, which is consistent with the Xue et al. study and suggests that aucubin protects chondrocytes from cell apoptosis which is often observed during OA progression.

Table 2 Quantitative results of Annexin V/PI test of aucubin

\begin{tabular}{|c|c|c|c|c|}
\hline & \multicolumn{4}{|c|}{ Percentage of cell (\%) } \\
\hline & Normal & Early apoptosis & Late apoptosisi & Necrosis \\
\hline Control & $89.7 \pm 9.5$ & $2.5 \pm 4.6$ & $5.0 \pm 3.8$ & $3.1 \pm 1.8$ \\
\hline $\mathrm{H}_{2} \mathrm{O}_{2}$ & $25.5 \pm 7.3^{a}$ & $29.4 \pm 4.2^{a}$ & $32.2 \pm 6.8^{a}$ & $14.3 \pm 7.6^{2}$ \\
\hline $\mathrm{H}_{2} \mathrm{O}_{2}+$ aucubin & $60.1 \pm 10.4^{a, b}$ & $15.4 \pm 5.3^{\mathrm{a}, \mathrm{b}}$ & $15.8 \pm 4.2^{\mathrm{a}, \mathrm{b}}$ & $10.7 \pm 5.3$ \\
\hline
\end{tabular}

Results shown represent mean \pm SD obtained from 5 repeated measurements. ${ }^{a} p<0.05$ compared with the control group. ${ }^{b} p<0.05$ compared with the $\mathrm{H}_{2} \mathrm{O}_{2}$ group 

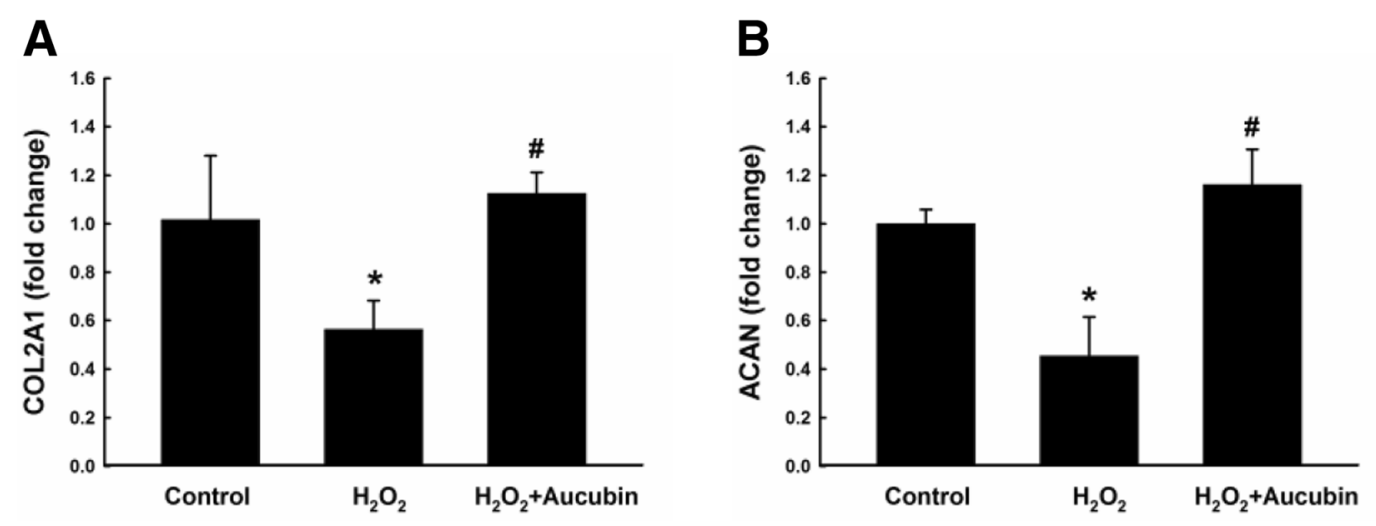

C

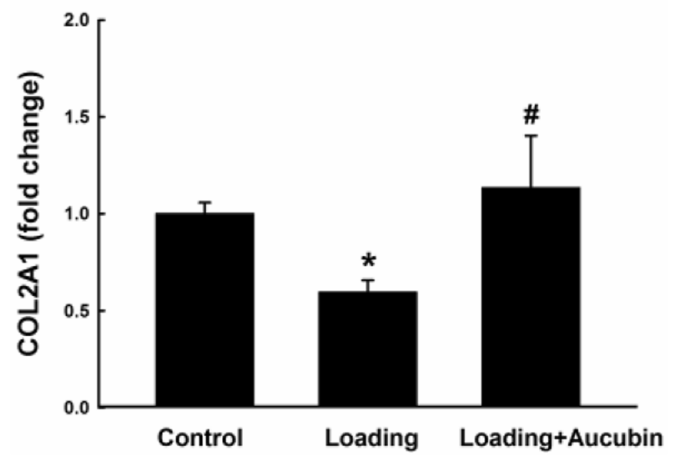

D

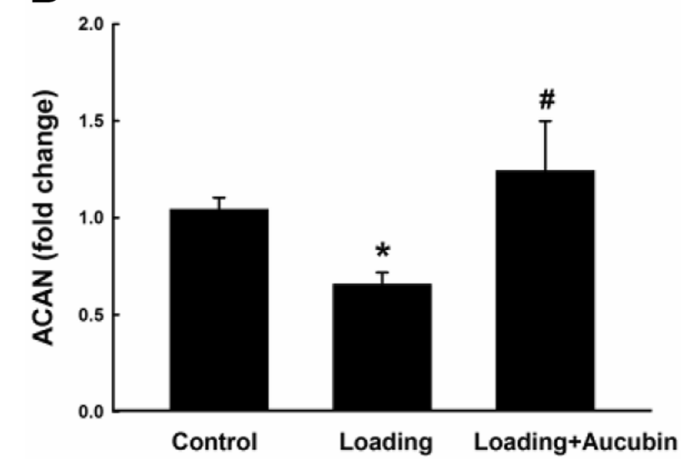

E

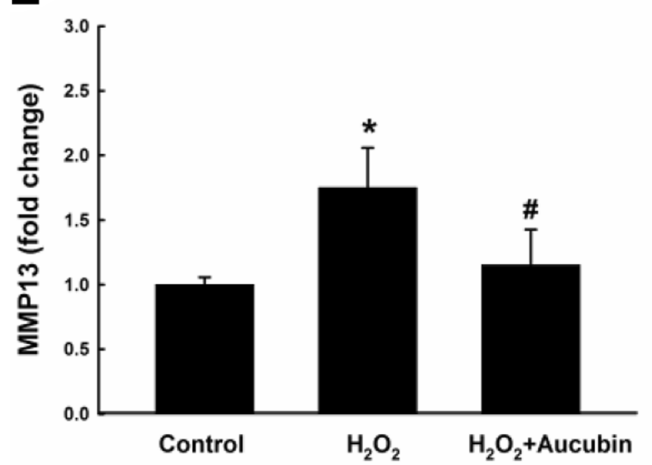

F
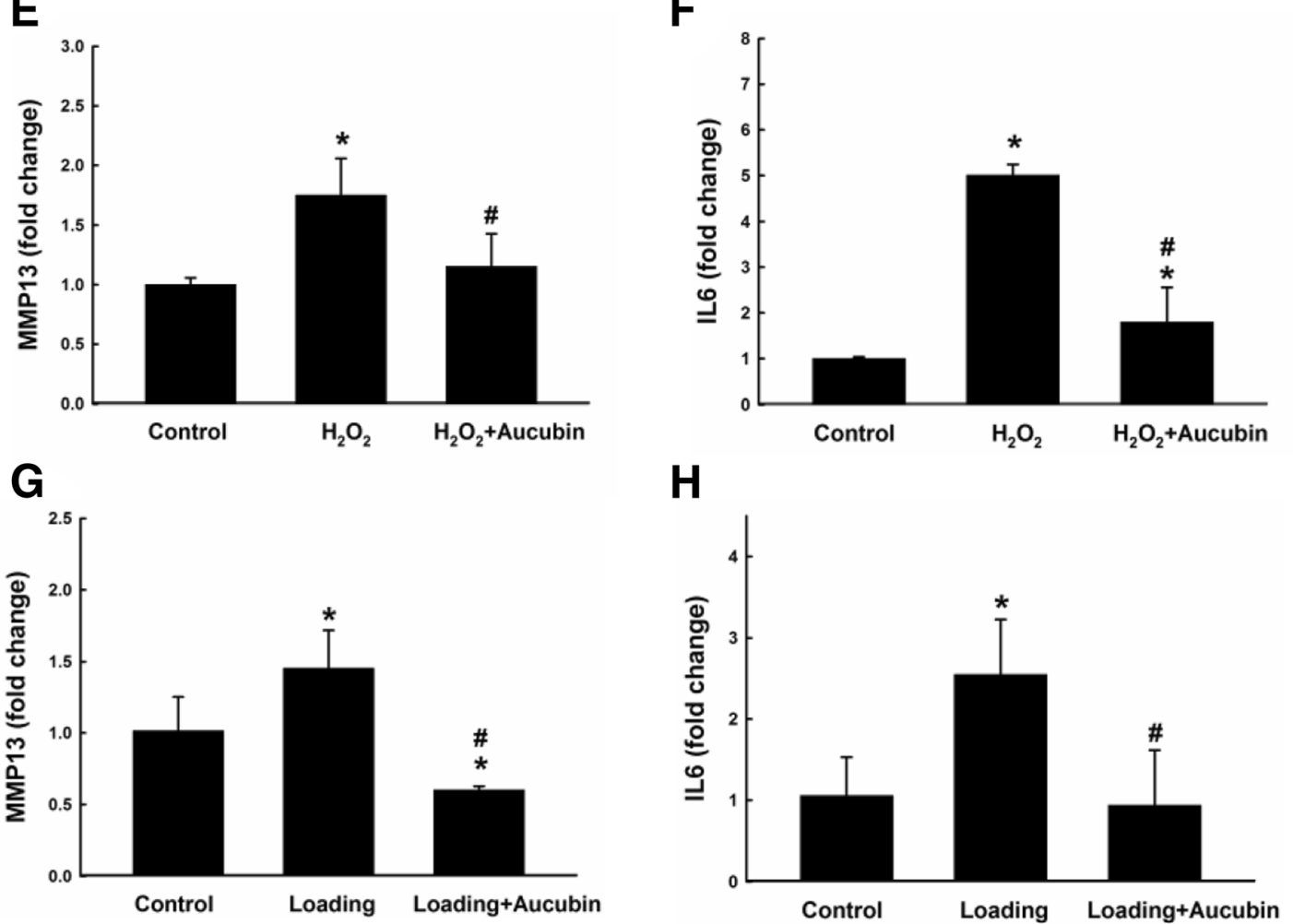

Fig. 6 (See legend on next page.) 
(See figure on previous page.)

Fig. 6 Gene expressions after stimulation. Gene expressions of COL2A1 (a, c), ACAN (b, d), MMP13 (e, g) and IL6 (f, h) were examined by RT-gPCR. Chondrocytes pretreated with $100 \mu \mathrm{M}$ of aucubin for $24 \mathrm{~h}$ were then stimulated with $1 \mathrm{mM} \mathrm{H}_{2} \mathrm{O}_{2}(\mathbf{a}$, bo e and $\mathbf{f})$ for 30 min, or 60 psi compression (c, $\mathbf{d}, \mathbf{g}$ and $\mathbf{h}$ ) for $24 \mathrm{~h}$. Data from five independent biological replicates each with three technical replicates are expressed as fold change compared to untreated control and shown represent mean \pm SD. Each target gene was normalized to GAPDH. ${ }^{*} p<0.05$ compared with the control group. ${ }^{\#} p<0.05$ compared with the $\mathrm{H}_{2} \mathrm{O}_{2}$ or loading group

Aggrecan and type II collagen are the main components of chondrocyte ECM and provide cartilage with compressive and tensile resistance, respectively. In the OA cartilage, a loss of proteoglycans and decrease in type II collagen has been observed, leading to defective integrity of the cartilage [29]. In this study, both $\mathrm{H}_{2} \mathrm{O}_{2}$ and compressive loading caused significant down-regulations of ACAN and
COL2A1, suggesting the stimulated chondrocytes were undergoing a degeneration process with weakened anabolic activity (Fig. 6). However, chondrocytes pretreated with aucubin showed a protective effect by reversing the $\mathrm{H}_{2} \mathrm{O}_{2}$ / compression induced down-regulation of both ACAN and COL2A1 genes, which may implicate that aucubin has a beneficial effect on chondrocyte ECM production.

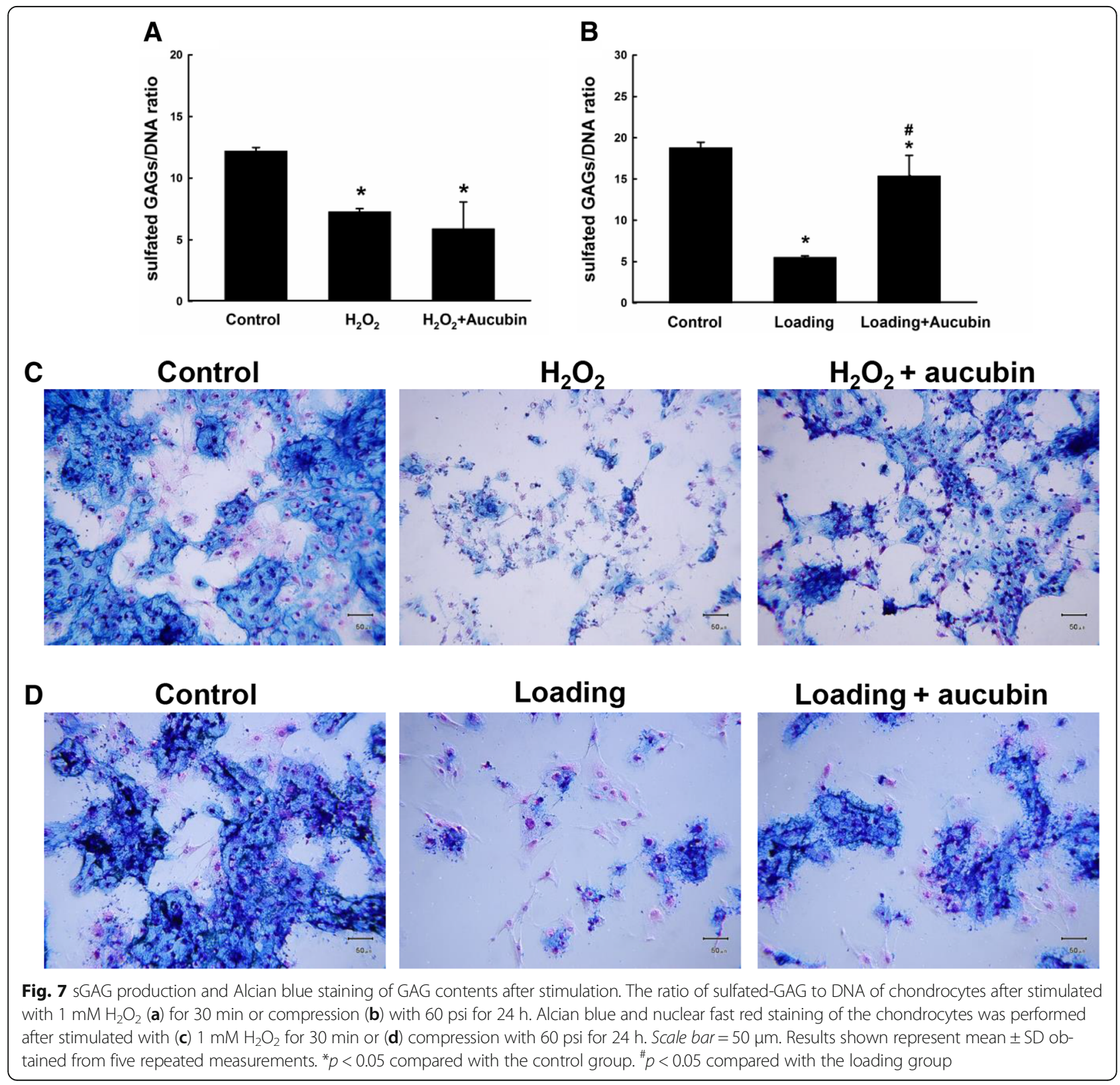


The expression of pro-inflammatory IL- 6 inhibits the synthesis of aggrecan and is highly elevated in OA cartilage [32]. IL-6 expression directly contributes to the inhibitory effect of aggrecan through the Notch receptor [33], mediating induction of MMP-13 expression [34]. MMP-13 is a catabolic factor for cartilage ECM metabolism, degrading proteoglycans and type II collagen $[35,36]$. Aucubin has been shown to inhibit TNFalpha and IL-6 production in antigen-stimulated rat basophilic leukemia-2H3 mast cells [37]. Aucubin also has been demonstrated to reverse the increased gene and protein expression of MMP13, iNOS and COX-2 induced by IL-1 $\beta$ stimulation in rat chondrocytes [38]. In this study, aucubin significantly reversed the elevated gene expression of IL6 and MMP13 genes in the $\mathrm{H}_{2} \mathrm{O}_{2}$ and compression stimulated chondrocytes, consistently demonstrating the anti-inflammatory and anti-catabolic properties of aucubin in chondrocytes (Fig. 6).

About the single reference gene of GAPDH, in the previous study we had examined the gene expression of porcine chondrocytes and used GAPDH as reference gene for RT-qPCR [28]. After analysis the GAPDH gene showed a consistent $\mathrm{Ct}$ value among groups with different treatment. In the present study, we used the same method to isolate the porcine chondrocytes and observed the same phenomenon of GAPDH expressions in RT-qPCR results. The $\mathrm{Ct}$ values of GAPDH did not show a noticeable difference among chondrocytes stimulated with compression, $\mathrm{H}_{2} \mathrm{O}_{2}$, Aucubin or in control group. We suggest that treatments used in the present study did not affect the expression of GAPDH gene, thus, we used GAPDH as the house keeping gene in this study.

In the degenerated cartilage, a decrease in the content of chondroitin sulfate results in a decrease of water content that affects the capability of cartilage to absorb the external stress [39]. In this study, $\mathrm{H}_{2} \mathrm{O}_{2}$ and compression stimulation significantly reduced the sGAG production activity and decreased sGAG content. Chondrocytes pretreated with aucubin succeeded to maintain its sGAG content after both $\mathrm{H}_{2} \mathrm{O}_{2}$ and compression stimulation, and recover its sGAG production activity after compression stimulation. However, aucubin failed to maintain the sGAG production activity after $\mathrm{H}_{2} \mathrm{O}_{2}$ stimulation (Fig. 7). According to these data, we suggest that the protective effects of aucubin may be overwhelmed by the oxidative stress of $\mathrm{H}_{2} \mathrm{O}_{2}$. The oxidative stress may lead to decrease the sGAG production activity of chondrocytes. Nevertheless, aucubin still has beneficial ability to maintain sGAG content of chondrocytes after $\mathrm{H}_{2} \mathrm{O}_{2}$ and compression stimulation.

\section{Conclusions}

This study demonstrated that aucubin can reduces ROS production, caspase-3 activity, and cell apoptosis.
Aucubin can protect porcine chondrocytes from $\mathrm{H}_{2} \mathrm{O}_{2}$ and compression-induced dysregulation of COL2A1, ACAN, IL6 and MMP13 gene expressions. Additionally, aucubin helps porcine chondrocytes maintain sGAG contents after $\mathrm{H}_{2} \mathrm{O}_{2}$ and compressive stimulation. These results indicated that aucubin has protective effects in an osteoarthritic chondrocyte model induced by $\mathrm{H}_{2} \mathrm{O}_{2}$ and mechanical stimulus.

\section{Abbreviations \\ ADAMTS: A disintegrin and metalloproteinase with thrombospondin motifs DMEM: Dulbecco's modified eagle's medium; ECM: Extracellular matrix; GAPDH: Glyceraldehyde-3-phosphate dehydrogenase; IL-6: Interlukin-6; LDH: Lactate dehydrogenase; MMP13: Matrix metalloproteinase 13; OA: Osteoarthritis; PBS: Phosphate buffered saline; ROS: Reactive oxygen species; RT-qPCR: Reverse transcription quantitative polymerase chain reaction; sGAG: Sulfated-glycosaminoglycan}

\section{Acknowledgements}

The authors wish to thank National Taiwan University academic writing education center for professional language editing.

\section{Funding}

This study was financially supported by National Science Council grant No. 100-2313-B-002-049-MY2. The funders had no role in study design, data collection and analysis, decision to publish, or preparation of the manuscript.

\section{Availability of data and materials}

The datasets during and/or analysed during the current study available from the corresponding author on reasonable request.

\section{Authors' contributions}

$\mathrm{ICY}$ and $\mathrm{FHL}$ conceived the research. ICY and FHL designed this work. FHL and $\mathrm{CHH}$ contributed new reagents and analytical tools. ICY performed the experiment. ICY, STC and YJS analyzed results. ICY, STC, HCL, YSC and FHL wrote the manuscript and revising it critically for important intellectual content. All authors read and approved the final manuscript.

\section{Competing interests}

The authors declare that they have no competing interests.

Consent for publication

Not applicable.

\section{Ethics approval and consent to participate}

The isolation and use of porcine chondrocytes were approved by the Animal Experimentation Ethics Committee of National Taiwan University Hospital.

\section{Author details}

Institute of Biomedical Engineering, National Taiwan University, No. 49, Fanglan Rd, Taipei 10672, Taiwan. ${ }^{2}$ Institute of Pharmacology, College of Medicine, National Taiwan University, No. 1, Sec. 1, Ren-Ai Rd, Taipei 10051, Taiwan. ${ }^{3}$ Institute of Biomedical Engineering and Nanomedicine, National Health Research Institute, No. 35, Keyan Rd, Miaoli 35053, Taiwan.

${ }^{4}$ Department of Orthopaedic Surgery, Taiwan Adventist Hospital, No. 424 Sec. 2, Bade Rd, Taipei 10556, Taiwan. '5epartment of Orthopaedic Surgery, National Taiwan University Hospital, No.7, Chung Shan S. Rd, Taipei 10002, Taiwan.

Received: 21 April 2016 Accepted: 13 January 2017

Published online: 02 February 2017

\section{References}

1. Dillon CF, Rasch EK, Gu Q, Hirsch R. Prevalence of knee osteoarthritis in the United States: arthritis data from the Third National Health and Nutrition Examination Survey 1991-94. J Rheumatol. 2006;33(1):2271-9.

2. Scotece M, Mobasheri A. Leptin in osteoarthritis: focus on articular cartilage and chondrocytes. Life Sci. 2015;1:75-8. 
3. Brosseau L, Rahman P, Toupin-April K, Poitras S, King J, De Angelis G, Loew $L$, et al. A systematic critical appraisal for non-pharmacological management of osteoarthritis using the appraisal of guidelines research and evaluation II instrument. PLoS One. 2014:9(1):e82986.

4. Jeong YJ, Kim I, Cho JH, Park DW, Kwon JE, Jung MW, et al. Anti-Osteoarthritic Effects of the Litsea japonica fruit in a rat model of osteoarthritis induced by monosodium iodoacetate. PLoS One. 2015;10(8):e0134856.

5. Heidari B. Knee osteoarthritis prevalence, risk factors, pathogenesis and features: part I. Caspian J Intern Med. 2011;2(2):205-12.

6. Felson DT. Developments in the clinical understanding of osteoarthritis. Arthritis Res Ther. 2009;11(1):203.

7. Li Y, Xu L. Advances in understanding cartilage remodeling. F1000Res. 2015; 4(F1000 Faculty Rev):642.

8. Kim H, Kang D, Cho Y, Kim JH. Epigenetic regulation of chondrocyte catabolism and anabolism in osteoarthritis. Mol Cells. 2015;38(8):677-84

9. Kapoor M, Martel-Pelletier J, Lajeunesse D, Pelletier JP, Fahmi H. Role of proinflammatory cytokines in the pathophysiology of osteoarthritis. Nat Rev Rheumatol. 2011;7(1):33-42.

10. Clouet J, Vinatier C, Merceron C, Pot-vaucel M, Maugars Y, Weiss P, et al. From osteoarthritis treatments to future regenerative therapies for cartilage. Drug Discov Today. 2009;14(19-20):913-25.

11. Musumeci G, Castrogiovanni P, Trovato FM, Weinberg AM, Al-Wasiyah MK, Alqahtani $\mathrm{MH}$, et al. Biomarkers of chondrocyte apoptosis and autophagy in osteoarthritis. Int J Mol Sci. 2015;16(9):20560-75.

12. Yamazaki K, Fukuda K, Matsukawa M, Hara F, Matsushita T, Yamamoto N, et al. Cyclic tensile stretch loaded on bovine chondrocytes causes depolymerization of hyaluronan: involvement of reactive oxygen species. Arthritis Rheum. 2003;48(11):3151-8

13. Kurz B, Lemke A, Kehn M, Domm C, Patwari P, Frank EH, et al. Influence of tissue maturation and antioxidants on the apoptotic response of articular cartilage after injurious compression. Arthritis Rheum. 2004;50(1):123-30.

14. Gabay O, Hall DJ, Berenbaum F, Henrotin Y, Sanchez C. Osteoarthritis and obesity: experimental models. Joint Bone Spine. 2008;75(6):675-9.

15. Liu SC, Lee HP, Hung CY, Tsai CH, Li TM, Tang CH. Berberine attenuates CCN2-induced IL-1 beta expression and prevents cartilage degradation in a rat model of osteoarthritis. Toxicol Appl Pharmacol. 2015;289(1):20-9.

16. Shen C, Cai GQ, Peng JP, Chen XD. Autophagy protects chondrocytes from glucocorticoids-induced apoptosis via ROS/Akt/FOXO3 signaling. Osteoarthritis Cartilage. 2015;23(12):2279-87.

17. Courties A, Gualillo O, Berenbaum F, Sellam J. Metabolic stress-induced joint inflammation and osteoarthritis. Osteoarthritis Cartilage. 2015; 23(11):1955-65.

18. Riedl SJ, Shi Y. Molecular mechanisms of caspase regulation during apoptosis. Nat Rev Mol Cell Biol. 2004;5(11):897-907.

19. Farooq SM, Boppana NB, Devarajan AC, Sekaran SD, Shankar EM, Li C, et al. C-phycocyanin confers protection against oxalate-mediated oxidative stress and mitochondrial dysfunctions in MDCK cells. PLoS One. 2014;9(4):e93056.

20. Cheng YH, Yang SH, Liu CC, Gefen A, Lin FH. Thermosensitive hydrogel made of ferulic acid-gelatin and chitosan glycerophosphate. Carbohydr Polym. 2013;92(2):1512-9.

21. Chang LM, Yun HS, Kim YS, Ahn JW. Aucubin: potential antidote for alphaamanitin poisoning. J Toxicol Clin Toxicol. 1984;22(1):77-85.

22. Li Y, Sato T, Metori K, Koike $K$, Che QM, Takahashi S. The promoting effects of geniposidic acid and aucubin in Eucommia ulmoides Oliver leaves on collagen synthesis. Biol Pharm Bull. 1998;21(12):1306-10.

23. Chang IM, Ryu JC, Park YC, Yun HS, Yang KH. Protective activities of aucubin against carbon tetrachloride-induced liver damage in mice. Drug Chem Toxicol. 1983;6(5):443-53.

24. Chang IM. Liver-protective activities of aucubin derived from traditional oriental medicine. Res Commun Mol Pathol Pharmacol. 1998;102(2):189-204.

25. Jin L, Xue HY, Jin LJ, Li SY, Xu YP. Antioxidant and pancreas-protective effect of aucubin on rats with streptozotocin-induced diabetes. Eur J Pharmacol. 2008;582(1-3):162-7.

26. Xue HY, Gao GZ, Lin QY, Jin LJ, Xu YP. Protective effects of aucubin on $\mathrm{H}_{2} \mathrm{O}_{2}$-induced apoptosis in PC12 cells. Phytother Res. 2012;26(3):369-74.

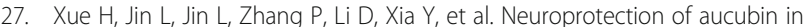
primary diabetic encephalopathy. Sci China C Life Sci. 2008;51(6):495-502.

28. Chang CH, Hsu YM, Chen YC, Lin FH, Sadhasivam S, Loo ST, et al. Antiinflammatory effects of hydrophilic and lipophilic statins with hyaluronic acid against LPS-induced inflammation in porcine articular chondrocytes. J Orthop Res. 2014;32(4):557-65.
29. Cheng YH, Yang SH, Lin FH. Thermosensitive chitosan-gelatin-glycerol phosphate hydrogel as a controlled release system of ferulic acid for nucleus pulposus regeneration. Biomaterials. 2011;32(29):6953-61.

30. Park H, Temenoff JS, Holland TA, Tabata Y, Mikos AG. Delivery of TGF-beta1 and chondrocytes via injectable, biodegradable hydrogels for cartilage tissue engineering applications. Biomaterials. 2005;26(34):7095-103.

31. Ho JN, Lee YH, Park JS, Jun WJ, Kim HK, Hong BS, et al. Protective effects of aucubin isolated from Eucommia ulmoides against UVB-induced oxidative stress in human skin fibroblasts. Biol Pharm Bull. 2005;28(7):1244-8.

32. Wang P, Guan PP, Guo C, Zhu F, Konstantopoulos K, Wang ZY. Fluid shear stress-induced osteoarthritis: roles of cyclooxygenase-2 and its metabolic products in inducing the expression of proinflammatory cytokines and matrix metalloproteinases. FASEB J. 2013;27(12):4664-77.

33. Zanotti S, Canalis E. Interleukin 6 mediates selected effects of Notch in chondrocytes. Osteoarthritis Cartilage. 2013;21(11):1766-73.

34. Ashraf S, Mapp PI, Burston J, Bennett AJ, Chapman V, Walsh DA. Augmented pain behavioural responses to intra-articular injection of nerve growth factor in two animal models of osteoarthritis. Ann Rheum Dis. 2014; 73(9):1710-8.

35. Halbwirth F, Niculescu-Morzsa E, Zwickl H, Bauer C, Nehrer S. Mechanostimulation changes the catabolic phenotype of human dedifferentiated osteoarthritic chondrocytes. Knee Surg Sports Traumatol Arthrosc. 2015;23(1):104-11.

36. Enochson L, Stenberg J, Brittberg M, Lindahl A. GDF5 reduces MMP13 expression in human chondrocytes via DKK1 mediated canonical Wnt signaling inhibition. Osteoarthritis Cartilage. 2014;22(4):566-77.

37. Jeong HJ, Koo HN, Na HJ, Kim MS, Hong SH, Eom JW, et al. Inhibition of TNF-alpha and IL-6 production by Aucubin through blockade of NF-kappaB activation RBL-2H3 mast cells. Cytokine. 2002;18(5):252-9.

38. Wang SN, Xie GP, Qin CH, Chen YR, Zhang KR, Li X, et al. Aucubin prevents interleukin-1 beta induced inflammation and cartilage matrix degradation via inhibition of NF-KB signaling pathway in rat articular chondrocytes. Int Immunopharmacol. 2015;24(2):408-15.

39. Vynios DH. Metabolism of cartilage proteoglycans in health and disease. Biomed Res Int. 2014:2014:452315.

\section{Submit your next manuscript to BioMed Central and we will help you at every step:}

- We accept pre-submission inquiries

- Our selector tool helps you to find the most relevant journal

- We provide round the clock customer support

- Convenient online submission

- Thorough peer review

- Inclusion in PubMed and all major indexing services

- Maximum visibility for your research

Submit your manuscript at www.biomedcentral.com/submit
) Biomed Central 